\title{
Tenesmus und Strangurie bei einem Rind infolge einer Zyste in der Harnblasenwand. Ein Fallbericht
}

\author{
Bruderer, A ; de Brot, S ; Nuss, Karl
}

\begin{abstract}
Ein 10 Monate altes, weibliches Braunviehrind zeigte Pollakisurie, Strangurie und Tenesmus. Ultrasonographisch und endoskopisch ließ sich ein zystisches Gebilde am Harnblasenpol darstellen. Das Rind wurde unter Vollnarkose in Rückenlage operiert und die Zubildung zusammen mit dem Harnblasenpol reseziert. Die histologische Untersuchung ergab den Befund einer von Urothel ausgekleideten Zyste. Neun Tage nach der Operation hatte sich der Harnabsatz so weit gebessert, dass das Rind aus der Klinik entlassen werden konnte. Laut telefonischer Auskunft des Besitzers war es 6 Monate nach der Entlassung klinisch unauffällig.
\end{abstract}

DOI: https://doi.org/10.1055/s-0038-1623184

Other titles: Tenesmus and stranguria in a heifer caused by a urachal cyst in the bladder wall

Posted at the Zurich Open Repository and Archive, University of Zurich

ZORA URL: https://doi.org/10.5167/uzh-89166

Journal Article

Accepted Version

Originally published at:

Bruderer, A; de Brot, S; Nuss, Karl (2013). Tenesmus und Strangurie bei einem Rind infolge einer Zyste in der Harnblasenwand. Ein Fallbericht. Tierärztliche Praxis. Ausgabe G, Grosstiere/Nutztiere, 41(G):326-329.

DOI: https://doi.org/10.1055/s-0038-1623184 
1 Tenesmus und Strangurie bei einem Rind infolge einer Zyste in der Harnblasenwand

$2 \quad$ Fallbericht

3

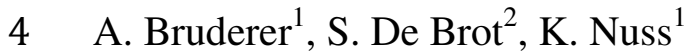

5

6 Aus der ${ }^{1}$ Abteilung für Nutztierchirurgie des Departementes für Nutztiere und dem ${ }^{2}$ Institut

7 für Veterinärpathologie der Vetsuisse-Fakultät Zürich

8

9 Korrespondenzadresse:

10

11 Karl Nuss

12 Prof. Dr. med. vet., Dipl. ECVS, Dipl. ECBHM

13 Departement für Nutztiere

14 Vetsuisse-Fakultät der Universität Zürich

15 Winterthurerstrasse 260

$16 \mathrm{CH}-8057$ Zürich, Schweiz

17 Tel: +41-44-6359031

18 Fax: +41-44-6358904

19 knuss@vetclinics.uzh.ch

20

21 
24 Schlüsselwörter

25 Rind, Tenesmus, Harnblase, Urachuszyste, Operation

\section{Zusammenfassung}

28 Ein zehn Monate altes Braunviehrind zeigte Pollakisurie, Strangurie und Tenesmus. 29 Ultrasonographisch und endoskopisch konnte ein zystisches Gebilde am Harnblasenpol 30 dargestellt werden. Das Rind wurde unter Vollnarkose in Rückenlage operiert und die 31 Zubildung zusammen mit dem Harnblasenpol reseziert. Die histologische Untersuchung ergab den Befund einer von Urothel ausgekleideten Zyste. Neun Tage nach der Operation hatte sich der Harnabsatz soweit gebessert, dass das Rind nach Hause entlassen werden konnte; es war bei einer telefonischen Nachkontrolle nach sechs Monaten symptomlos.

\section{Key words}

Heifer, tenesmus, urinary bladder, urachal cyst, surgery

\section{Summary}

41 A 10-month-old Swiss Braunvieh heifer was referred to our clinic because of pollakiuria, 42 stranguria and tenesmus. Ultrasonography and endoscopy revealed a cyst-like lesion at the 43 apex of the urinary bladder. The apex of the bladder was surgically removed with the patient 44 in dorsal recumbency under general anaesthesia. Histological examination revealed a cyst 45 lined with urothelium in the wall of the urinary bladder. The heifer was discharged nine days 46 after surgery and was healthy six months later. 


\section{$51 \quad$ Einleitung}

52 Das Leitsymptom von Urachusanomalien ist eine Störung des Harnabsatzes. Diese erfolgt entweder aufgrund eines ständigen Reizzustandes der Harnblase, durch Zug am Blasenpol durch die bestehende Verbindung zum Nabel oder durch Verklebungen mit anderen Organen der Bauchhöhle infolge einer Entzündung. Dieser Bericht handelt von einer Urachusanomalie, bei der weder Veränderungen am äußeren Nabel, noch ein persistierender Urachus, noch Verklebungen vorlagen.

\section{Anamnese und Klinische Untersuchung}

Das knapp zehn Monate alte, weibliche, $230 \mathrm{~kg}$ schwere Braunviehrind aus Laufstallhaltung hatte seit Geburt Pollakisurie und Strangurie gezeigt. Der äußere Nabel war allerdings immer unauffällig gewesen. Des Weiteren war bei dem Tier seit längerem dünnflüssiger Kot beobachtet worden.

Bei der Klinischen Allgemeinuntersuchung zeigte das Rind eine leicht erhöhte Herzfrequenz (100/Minute). Atemfrequenz (28/Minute) und Körpertemperatur $\left(38,1^{\circ} \mathrm{C}\right)$ waren nicht verändert. Die sichtbaren Schleimhäute waren blassrosa, die Futteraufnahme war ungestört. Während einer dreitägigen Beobachtungs- und Untersuchungszeit konnten die Pollakisurie und die Strangurie bestätigt werden. Der mit einem Teststreifen (Combur-Test ${ }^{\circledR}$, F. Hoffmann-La Roche AG, Basel, Schweiz) untersuchte Harn war ohne besonderen Befund. Zusätzlich zeigte das Tier Tenesmus, wobei stets kleine Mengen von dünnflüssigem Kot ausgepresst wurden.

\section{Palpations-, Ultraschall- und Zystoskopiebefunde}

Im äusseren Nabel konnte ein weicher, bleistiftstarker Strang palpiert werden, der weder palpatorisch noch ultrasonographisch in die Bauchhöhle verfolgbar war. Transabdominal war die Harnblase nicht sichtbar, und es konnte auch keine Verbindung zwischen dem äußeren Nabel und der Harnblase dargestellt werden.

Daraufhin wurde eine transrektale Ultraschalluntersuchung (MYLAB ${ }^{\text {TMOne Vet, SV3513 }}$ Sonde, 10-5 MHz, Esaote, Genua, Italien) durchgeführt. Bei der im Becken liegenden Harnblase konnte am kranialen Pol ein zystisches Gebilde von ca. 4,5 x 3,5 cm Grösse dargestellt werden (Abb. 1). Der Inhalt dieses Gebildes war mehrheitlich anechogen, jedoch durchsetzt mit hyperechogenen, festen Anteilen. 
Ultraschall festgestellte Umfangsvermehrung am Blasenpol sichtbar. Sie ragte kugelförmig, vom Harnblasenpol ausgehend, in das Lumen der Harnblase hinein. Sie wies eine glatte, mit Schleimhaut überzogene Oberfläche auf (Abb. 2). Das übrige Harnblasenlumen war ohne besonderen Befund.

\section{Vorbereitung und Anästhesie}

Das Rind wurde für 18 Stunden gefastet und mit Xylazin $(0,1 \mathrm{mg} / \mathrm{kg}$ KM i.m., Streuli Pharma AG, Uznach, Schweiz) sediert. Danach wurde die Narkose über einen intravenösen Verweilkatheter mit Ketamin (Narketan ${ }^{\circledR} 10,2,0 \mathrm{mg} / \mathrm{kg}$ KM i.v., Vétoquinol AG, Ittingen, Schweiz) eingeleitet. Nach der Intubation mit einem Tubus der Grösse 14.0 wurde die Allgemeinanästhesie mittels Isofluran (Isoflo®, 1,5-2,5\%, Abbott AG, Baar, Schweiz) und einer Ketamin-Dauertropfinfusion (Narketan ${ }^{\circledR} 10,0,6 \mathrm{mg} / \mathrm{kg} / \mathrm{h}$ KM i.v., Vétoquinol AG, Ittingen, Schweiz) mittels Infusomat (SYRAMED ${ }^{\mathrm{TM}}, \mu$ SP 6000, arcomed AG, Regensdorf, Schweiz) aufrecht erhalten.

Zusätzlich erhielt das Tier Flunixin meglumin (Fluniximin, 1,1mg/kg KM i.v., Dr. E. Graeub AG, Bern, Schweiz), Benzylpenicillinum procainum (Procacillin®, 1,2Mio IE/kg KM i.m., Veterinaria AG, Pfäffikon SZ, Schweiz), Vitamine A, $D_{3}$ und E (AquaVit ${ }^{\circledR}, 250 ` 000$ IE Retinolazetat, 125'000 IE Cholecalciferol und 125mg Tocopherolazetat pro Kalb, Werner Stricker AG, Zollikofen, Schweiz), sowie Selen und Vitamin E (Tocoselenit ad us vet., 0,2mg Natriumselenit und 5mg Vitamin E pro kg KM s.c., Dr. E. Graeub AB, Bern, Schweiz). Für die Operation wurde das Rind in Rückenlage auf einem Operationstisch positioniert.

\section{Operationsprozedere und intraoperative Befunde}

Der Hautschnitt erfolgte von $10 \mathrm{~cm}$ kaudal des Nabels bis unmittelbar kranial des Beckens, unter Schonung des Eutergewebes, in der Medianen. Die Bauchhöhle wurde in der Linea alba eröffnet und zunächst exploriert. Die Harnblase war klein. Am Harnblasenpol befand sich eine leicht eindrückbare, runde Zyste, welche mit den beiden verkürzten Nabelarterien verwachsen und nur schlecht aus der Bauchhöhle vorzulagern war. Die Nabelarterien wurden freipräpariert, proximal ligiert und abgesetzt. Zwischen Harnblase und Uterus bestanden Verklebungen, die stumpf-scharf gelöst wurden. Nach Anbringen von Haltefäden in der Harnblasenwand wurden Darmklemmen am Harnblasenpol angesetzt (Abb. 3). Die Zyste wurde unter Absetzen des Blasenpols exzidiert und danach die Harnblase zweischichtig nach Kürschner und Cushing mit einem monofilen Nahtmaterial (BIOSYN ${ }^{\text {TM }}$, USP 2.0 CONVIDIEN, Dublin, Irland) verschlossen. 
119 Nach Lavage der Bauchhöhle wurden die Bauchhöhle und die Bauchdecke in mehreren 120 Schichten adaptiert.

121 Postoperativ konnte die korrekte Entfernung der Zyste am Harnblasenpol am Präparat 122 verifiziert werden. Der Zysteninhalt war flockig eitrig und hatte keine direkte Verbindung zu 123 den Nabelarterien.

124 Zur Nachbehandlung erhielt das Rind für zwei weitere Tage Fluniximin und Procacillin in der 125 oben angegebenen Dosierung. Da das Rind postoperativ eine erhöhte Körpertemperatur 126 aufwies, wurde die Antibiose auf Amoxicillin (Clamoxyl, 7 mg/kg KM i.m., Pfizer AG, 127 Zürich, Schweiz) und schließlich auf Cefquinom (Cobactan® 2.5\%, 1mg/kg KM i.m., 128 Veterinaria AG, Pfäffikon SZ, Schweiz) umgestellt. Neun Tage nach der Operation wurde das 129 Rind mit gutem Allgemeinzustand, fieberfrei und mit sich normalisierendem Kot- und 130 Harnabsatz nach Hause entlassen. Sechs Monate postoperativ zeigte es laut telefonischer 131 Befragung des Besitzers keine Harnabsatzstörungen mehr und entwickelte sich entsprechend 132 wie seine Artgenossen.

\section{Histologische Untersuchung der Zyste}

135 Bei der exzidierten Veränderung handelte es sich um ein zystisches Gebilde mit einer 136 Wanddicke von 2 bis $3 \mathrm{~mm}$. Die Zystenwand wurde von einer inneren epithelialen und einer 137 äußeren bindegewebigen Schicht gebildet. Beim Epithel handelte es sich um normales 138 Übergangsepithel (Urothel, Abb. 4). Das darüberliegende Bindegewebe war locker 139 angeordnet und gefäßreich. Der in die Bauchhöhle ragende Teil der Zyste war mit 140 abgeflachtem, einschichtigem Epithel (Serosa) überzogen. Zur Harnblase hin folgte auf die 141 Bindegewebsschicht direkt das Urothel der Harnblase. Im Bereich der abgehenden 142 Nabelarterien war glattes Muskelgewebe vorhanden, wohingegen in der restlichen Zyste kein 143 muskuläres Gewebe angesprochen werden konnte. Der Zysteninhalt bestand aus viel 144 nekrotischem Material und einem ausgedehnten Bakterienrasen, möglicherweise bedingt 145 durch eine vorangegangene Infektion. Histologisch wurden keine Hinweise auf neoplastische 146 Veränderungen gefunden.

\section{Diskussion}

149 Die embryonale Ausbildung des Urachus erfolgt, wenn sich die Harnblase aus der 150 Nabelregion ins Becken verlagert. Da die Harnblase am Nabel mit dem Allantoisgang 151 verbunden ist, ist es schwierig zu bestimmen, welche Teile des Urachus vom Allantoisgang 152 und welche von der in die Länge gezogenen Harnblase stammen (7). Zum Zeitpunkt der 
153 Geburt zieht sich der Urachus in die Bauchhöhle zurück, wo er als geschlängeltes ca. $2-5 \mathrm{~cm}$

154 langes Band am Blasenpol verbleibt und sich später als Blasennabel darstellt (6). Histologisch 155 besteht der Urachus aus dem Übergangsepithel (Urothel), einer Verbindungsschicht und einer 156 äußeren Muskelschicht mit Serosaüberzug (2). Die verhältnismäßig dicke Adventitia der 157 Harnblase geht nicht in den Urachus über. Daher kommt es am Übergang von Harnblase zum 158 Urachus zu einer rundlichen Aussparung in der Wand. Diese Öffnung kann den 159 Schwachpunkt bilden, der für die Bildung von Urachusdivertikeln und -zysten 160 prädisponierend ist. Bei der im vorliegenden Fall beschriebenen Zyste handelte es sich 161 entweder um ein Überbleibsel des Urachus, das sich primär infizierte und abkapselte, oder 162 da sie keine Muskelschicht aufwies - um ein Überbleibsel des Allantoisganges. Eine andere 163 Möglichkeit ist die Bildung einer Zyste im Bereich des Blasenpols, die sich sekundär 164 infizierte, wobei der Infektionsweg in den seltensten Fällen nachvollzogen werden kann (7).

$165 \mathrm{Zu}$ den Urachusanomalien zählen weiterhin persistierender und patenter Urachus, 166 vesikourethrales Divertikel, Urachussinus und Urachusabszesse (5, 9). Im vorliegenden Fall 167 lag in der Zyste ein eitriges Sekret vor, aber es handelte sich nicht um einen Abszess, da die 168 Zyste von Urothel ausgekleidet war. Zudem war die Zyste in die Wand der Harnblase 169 integriert, das heißt, sie reichte sowohl in das Harnblasenlumen hinein als auch über die 170 Harnblasenwand nach außen.

171 Allen Urachuserkrankungen gemeinsam ist das Symptom der Pollakisurie, wobei es bei 172 sekundärer Zystitis zur Strangurie kommt (6). Die Ursache der vermehrten Frequenz des 173 Harnabsatzes besteht vor allem darin, dass die Urachusreste die Harnblase daran hindern, sich 174 vollständig zu entleeren, was zu vermehrtem Harndrang führt $(1,8)$. Tenesmus tritt vor allem 175 im Zusammenhang mit lokalen Reizzuständen im rektovaginalen oder analen Bereich auf, z.

176 B. bei Passagestörungen oder raumfordernden Prozesse im Beckenbereich. Andererseits 177 können auch eine schwere Enteritis oder Proktitis, sowie Tollwut und Aujeszkysche 178 Krankheit als Ursache in Frage kommen (4). Des Weiteren können Adenome und Fibrome 179 des Rektums Tenesmus hervorrufen (3). Auch Veränderungen im Bereich der 180 Harnröhrenmündung, wie ein erweitertes suburethrales Divertikulum, sind eine mögliche 181 Ursache (10). In dem hier vorgestellten Fall wurde die Harnblase durch die verkürzten Aa. 182 umbilicales an ihrer Ausdehnung über die Beckenhöhle hinaus gehindert, was vermutlich die 183 Pollakisurie verursachte. Der Tenesmus wurde wahrscheinlich durch die Zyste, die einen 184 ständigen Druck auf das Rektum auslöste, verursacht. 


\section{Literatur}

1. Baird AN. Umbilical Surgery in Calves. Vet Clin North Am - Food Anim Pract 2008; 24 (3): 467-477.

2. Begg RC. The urachus its anatomy, histology and development. J Anat 1930; 64: 170-83.

3. Bertone AL. Neoplasms of the Bovine Gastrointestinal Tract. Vet Clin North Am - Food Anim Pract 1990; 6 (2): 515-24.

4. Dirksen, G: After-Mastdarmzwang, Mastdarmvorfall. In: Dirksen G, Gründer H-D, Stöber M, Hrsg. Innere Medizin und Chirurgie des Rindes, 4. Aufl. Berlin, Parey 2002; 545-548.

5. Lischer CJ, Iselin U, Steiner A. Ultrasonographic diagnosis of urachal cyst in three calves. J Am Vet Med Assoc 1994; 204 (11): 1801-1804.

6. Nuss K. Erkrankungen der inneren Nabelstrukturen beim Rind. Tierärztl Prax 2007; 35 (G): 149-156.

7. Scheye Th., Vanneuville G., Amara B., Francannet Ph., Dechelotte P., Campagne D. Anatomic basis of pathology of the urachus. Surg Radiol Anat 1994; 16: 135-141.

8. Steiner A, Baumann D, Fluckiger M. Urachusabszess ohne pathologische Veränderungen des extraabdominalen Nabels bei einem Rind. Tierärztl Prax 1988; 16 (1): 33-36.

9. Trent AM, Smith DF. Pollakiuria due to urachal abscesses in two heifers. J Am Vet Med Assoc 1984; 15; 184 (8): 984-986.

10. Vogel SR, Dore E, Breteau G, Desrochers A, Babkine M, Nichols S. Congenital enlargement of the suburethral diverticulum in a Holstein calf. Can Vet J 2011; 52 (2): 173-176.

11. Wieland, M. Nabelerkrankungen des Kalbes: Formen, Symptomatik, Therapie und 
Abb. 2: Rind von Abbildung 1. Endoskopische Darstellung der Harnblase mit rundlicher, in das Lumen der Harnblase ragender Zubildung am Harnblasenpol.

Fig. 2: Endoscopic view of the urinary bladder of the heifer in Fig. 1 showing a round mass projecting into the lumen of the urinary bladder.

Abb. 3: Rind von Abbildung 1. Intraoperative Ansicht der rundlichen Masse am Harnblasenpol. Die Nabelarterien wurden ligiert und abgesetzt und Haltefäden wurden in der Harnblasenwand verankert. Die Darmklemme wurde zur Resektion des Harnblasenpols angelegt.

Fig. 3: Intraoperative view of a mass at the apex of the urinary bladder in the heifer in Fig. 1. The umbilical arteries have been ligated and severed, stay sutures have been placed in the bladder wall and a clamp has been placed proximal to the mass for resection.

Abb. 4 a und b) Rind von Abbildung 1. Histologie der Urachuszyste. a) Hämatoxylin-Eosin (HE), 1x.Die Zystenwand (W) wird von viel lockerem und gefäßreichem Bindegewebe gebildet und von einem mehrreihigem Übergangsepithel (Pfeilspitzen) ausgekleidet. Das Zystenlumen (L) ist mit viel nekrotischem Material $(\mathrm{N})$ und Bakterienrasen gefüllt. An der Außenseite wird die Zyste von einem einschichtigen Plattenepithel überzogen (Serosa = Mesothel) (Pfeile).

b) Bild des inneren Anteils der Zystenwand, Hämatoxylin-Eosin (HE), 20x. Zum Lumen (L) hin wird die Zyste von einem mehrreihigem Übergangsepithel (E) ausgekleidet. Die Zystenwand besteht aus viel lockerem und gefäßreichem Bindegewebe (B).

Figs. 4a and 4b; Histological examination of the specimen shown in Figs. 1 to 3.

a) The cyst wall (W) consists of loose vascular connective tissue and is lined on the inside by multi-layered transitional epithelium (urothelium) (arrow heads). There is a large clump of necrotic material $(\mathrm{N})$ covered with bacteria in the cyst lumen $(\mathrm{L})$. The outside of the cyst is lined by a single layer of squamous epithelium (serosa, mesothelium)(arrows). H\&E, x1. b) Close-up view of the inner part of the cyst wall showing loose vascular connective tissue (B) bordered on the inside by multi-layered transitional epithelium (E). H\&E, x20. 